\title{
Impacto da pandemia do Covid-19 na saúde dos idosos e intervenção da equipe de enfermagem
}

Impact of the Covid-19 pandemic on the health of the elderly and the intervention of the nursing team

Impacto de la pandemia Covid-19 en la salud de los ancianos y la intervención del equipo de enfermeira

Recebido: 20/10/2021 | Revisado: 25/10/2021 | Aceito: 26/10/2021 | Publicado: 29/10/2021

Thainá Oliveira Alves

ORCID: https://orcid.org/0000-0002-6128-6212 Faculdade Integrada Carajás, Brasil E-mail: thaina_samah@hotmail.com

Weslem Almeida Silva Nunes

ORCID: https://orcid.org/0000-0002-1960-2744 Faculdade Integrada Carajás, Brasil E-mail: wesley-nunes123@hotmail.com

Marcos Vinícios Ferreira dos Santos

ORCID: https://orcid.org/0000-0003-1335-1021

Faculdade Integrada Carajás, Brasil

E-mail: viniciosferreirasantos@hotmail.com

\begin{abstract}
Resumo
A pandemia da Covid-19 é reconhecida pela OMS como uma emergência internacional de saúde pública, o que revela vários aspectos da saúde, patogênese, epidemiologia e cuidados. As populações mais suscetíveis incluem idosos independentemente de seu estado de saúde, dentre outros, destaca-se a relevância de compreender o papel da enfermagem no contexto da saúde do idoso. O objetivo deste trabalho é discutir o impacto da pandemia mundial na saúde do idoso e a necessidade de uma intervenção profissional para manutenção da qualidade de vida. A busca de estudos realizou-se nas bases de dados NCBI/PubMed, SciELO, Google Acadêmico e Lilacs - Bireme. Para delimitação dos conteúdos foram utilizados critérios de inclusão: artigos disponíveis na integra, em português e inglês, no período de 2019 a 2021 com acesso gratuito e que tivessem pertinência com tema. A partir da análise dos nove artigos, demonstrou-se que os idosos enfrentam mais ameaças e desafios frente à realidade, pois as alterações fisiológicas causadas pelo processo de envelhecimento afetam diretamente sua função imunológica e possíveis condições de saúde subjacentes. E ainda, fatores temporais e patologias típicas, como demência, acidente vascular cerebral e fraturas, os idosos têm aumentado sua suscetibilidade à infecção e embolia, o que está relacionado à infecção atual causada pela pandemia. Concluiu-se que o enfermeiro é fundamental para promoção da saúde do idoso, e realização de ações para conscientização do idoso, pois, os impactos da pandemia os atingem em diversos aspectos.

Palavras-chave: Idoso; Enfermagem; Covid-19; Impactos; Saúde.
\end{abstract}

\begin{abstract}
The Covid-19 pandemic is recognized by the WHO as an international public health emergency, which reveals several aspects of health, pathogenesis, epidemiology and care. The most susceptible populations include elderly people regardless of their health status, among others, the importance of understanding the role of nursing in the context of elderly health is highlighted. The objective of this paper is to discuss the impact of the global pandemic on the health of the elderly and the need for professional intervention to maintain quality of life. The search for studies was carried out in the NCBI/PubMed, SciELO, Academic Google and Lilacs - Bireme databases. To delimit the contents, inclusion criteria were used: articles available in full, in Portuguese and English, from 2019 to 2021 with free access and that were relevant to the theme. From the analysis of the nine articles, it was shown that the elderly face more threats and challenges in face of reality, as the physiological changes caused by the aging process directly affect their immune function and possible underlying health conditions. In addition, temporal factors and typical pathologies such as dementia, stroke and fractures, the elderly have increased their susceptibility to infection and embolism, which is related to the current infection caused by the pandemic. It was concluded that nurses are essential for promoting the health of the elderly, and carrying out actions to raise awareness of the elderly, as the impacts of the pandemic affect them in several aspects.
\end{abstract}

Keywords: Elderly; Nursing; Covid-19; Impacts; Health. 


\begin{abstract}
Resumen
La pandemia de Covid-19 es reconocida por la OMS como una emergencia de salud pública internacional, lo que revela varios aspectos de salud, patogénesis, epidemiología y atención. Las poblaciones más susceptibles incluyen a las personas mayores independientemente de su estado de salud, entre otras, se destaca la importancia de comprender el papel de la enfermería en el contexto de la salud del anciano. El objetivo de este artículo es discutir el impacto de la pandemia global en la salud de las personas mayores y la necesidad de intervención profesional para mantener la calidad de vida. La búsqueda de estudios se realizó en las bases de datos NCBI / PubMed, SciELO, Academic Google y Lilacs - Bireme. Para delimitar los contenidos se utilizaron criterios de inclusión: artículos disponibles íntegramente, en portugués e inglés, de 2019 a 2021 con acceso gratuito y que fueran relevantes para la temática. A partir del análisis de los nueve artículos, se demostró que las personas mayores enfrentan más amenazas y desafíos ante la realidad, ya que los cambios fisiológicos provocados por el proceso de envejecimiento afectan directamente su función inmunológica y posibles condiciones de salud subyacentes. Además, factores temporales y patologías típicas como demencia, ictus y fracturas, los ancianos han aumentado su susceptibilidad a infecciones y embolias, lo que se relaciona con la infección actual provocada por la pandemia. Se concluyó que las enfermeras son fundamentales para promover la salud de las personas mayores y realizar acciones de sensibilización de las personas mayores, ya que los impactos de la pandemia las afectan en varios aspectos.
\end{abstract}

Palabras clave: Anciano; Enfermería; Covid-19; Impactos; Salud.

\title{
1. Introdução
}

A Severe Acute Respiratory Syndrome Coronavirus-2 (Sars-Cov-2) denominada de coronavírus é transmitido por gotículas (saliva, espirros, tosse ou expectoração) na boca e no nariz. Essas gotículas podem ficar suspensas no ar ou se espalhar por contato pessoal, como apertar ou tocar as mãos, tocar em objetos e objetos contaminados (Brasil, 2020a). O período de incubação do coronavírus (o tempo desde a infecção humana até o aparecimento dos sintomas da doença) pode variar de 1 a 14 dias, mas geralmente fica em torno de 5 dias. Os sinais e sintomas mais comuns de Covid-19 são: febre, tosse seca, dispneia, fadiga, fadiga, dor de garganta, dor de cabeça, perda do olfato e paladar (Batello et al., 2020).

Destaca-se que em decorrência da sua alta taxa de transmissão na população, a doença é enfrentada atualmente em diversos países do mundo, levando a Organização Mundial da Saúde (OMS) a declarar o surto como uma emergência de saúde pública de importância internacional (ESPII), o que foi posteriormente classificada como uma pandemia. Atualmente, é um dos principais problemas de saúde pública do mundo. Essa situação tem levado muitos países a adotarem medidas restritivas mais rígidas, como o isolamento horizontal e o distanciamento social, com o objetivo de reduzir e disseminar o vírus (Ornelli et al., 2020; Brasil, 2020b).

Diante da pandemia causada pela Covid-19, a população idosa tem se destacado por fazer parte do grupo de risco e, devido às alterações ocasionadas pelo envelhecimento, são mais suscetíveis ao coronavírus. Além disso, o risco de morrer de Covid-19 aumenta com a idade, especialmente entre pessoas mais velhas com doenças crônicas. Este evento também se deve ao envelhecimento imunológico, pois com o processo de envelhecimento, o sistema imunológico muda, aumentando a vulnerabilidade a doenças infecciosas e, no caso de idosos infectados com Covid-19, em muitos casos, o prognóstico de pacientes com doenças crônicas é desfavorável (Costa et al., 2020).

Nesse sentido, fica evidente a necessidade de observações diferenciadas para essa parcela da população. Os idosos precisam de mais atenção e proteção para não contrair Covid-19, por isso é necessário adotar medidas mais rígidas de higiene, distância e isolamento social, e evitar o contato próximo com pessoas infectadas pelo coronavírus, lavar as mãos com frequência e evitar abraços e beijos (Silva; Donoso; Barbosa, 2021).

Portanto, é importante enfatizar o papel dos profissionais de saúde na resposta à pandemia Covid-19, especialmente os profissionais de enfermagem, pois se destacam no trabalho e atuam na linha de frente para atendê-los e buscam incessantemente a promoção da saúde, e a manutenção da vida. Desta forma o objetivo deste trabalho é discutir o impacto da pandemia mundial na saúde do idoso e a necessidade de uma intervenção profissional para manutenção da qualidade de vida. 


\section{Metodologia}

Este trabalho utilizou-se da revisão integrativa da literatura para discutir o tema sobre o impacto da pandemia do Covid-19 na saúde dos idosos e intervenção da equipe de enfermagem. Foram selecionados critérios de inclusão e exclusão para delimitação dos trabalhos, como critério de inclusão foram selecionadas bases de dados para pesquisa: NCBI/PubMed (National Center for BiotechnologyInformation), SciELO (Scientific Eletronic Lirary Online), Google Acadêmico e Lilacs Bireme (Literatura Latino-Americana e do Caribe em Ciências da Saúde) (Estrela, 2018).

Outro fator está correlacionado ao período de publicação dos artigos foram analisados apenas aqueles publicados entre os anos de 2013 à 2021, foram ainda escolhidas palavras chave para pesquisa "Covid-19", "idoso", "enfermagem geriátrica", "intervenção", sendo aceitos apenas os artigos publicados ou traduzidos em português, a metodologia dos artigos analisados é de cunho: bibliográfico, integrativo, sistemático, literário, aplicação de questionários e estudos de campo (Pereira et al., 2018).

Por outro lado, também foram aplicados critérios de exclusão tais como: não análise de artigos publicados de 2012 abaixo, textos incompletos, textos publicados em outras línguas, revisões empíricas e publicadas em outras plataformas diversas das escolhidas para esta pesquisa.

\section{Resultados e Discussão}

Identificaram-se no total 43 publicações, após aplicação dos critérios de inclusão, foram excluídos 34 estudos identificados por meio de pesquisas em outras fontes de dados, permanecendo apenas 9 artigos analisados no tópico discussão deste artigos, distribuídos nas seguintes plataformas de pesquisa: PubMed, SciELO, Google Acadêmico (G.A) e Lilacs. Os resultados apresentados acima foram dispostos no Fluxograma 1.

Fluxograma 1: Fluxograma das etapas de inclusão e exclusão dos artigos.
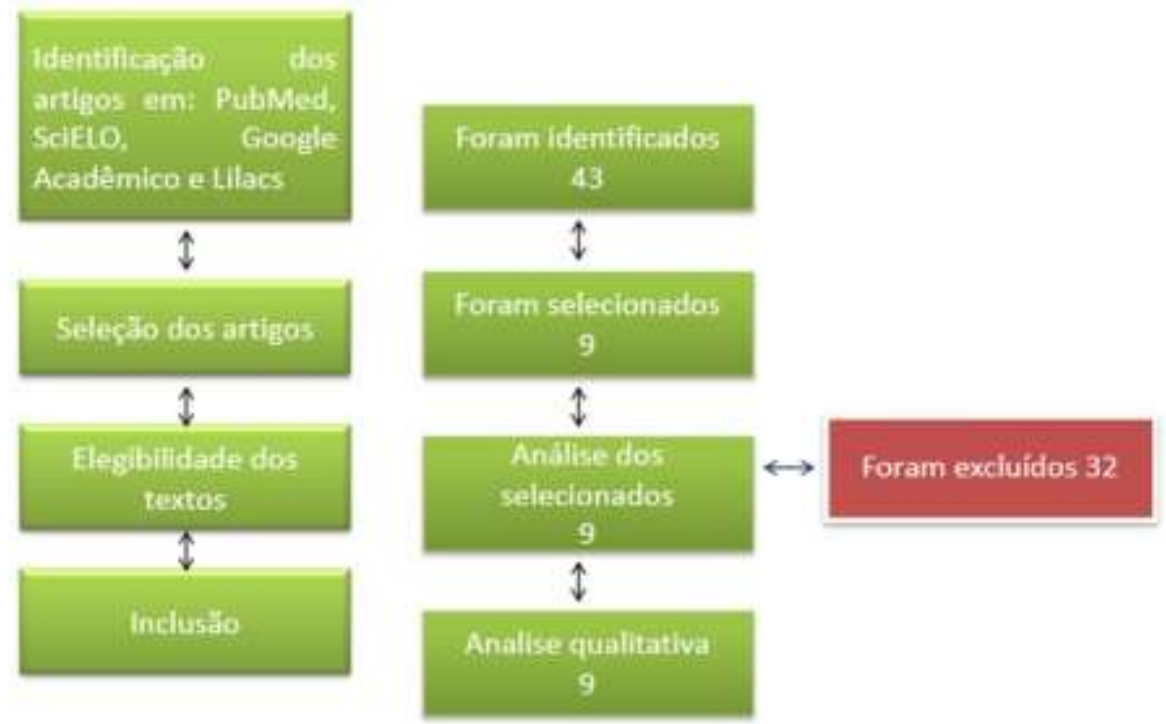

Fonte: Autores (2021).

Após a análise dos artigos foram selecionados 9 estudos produções para integrar este artigo de revisão. A Tabela 1 apresenta os textos escolhidos e sua distribuição por autoria, ano de publicação, título, revista e base de dados dos estudos selecionados. 
Quadro 1: Estudos selecionados segundo autoria, ano de publicação, título, revista e base de dados dos estudos selecionados.

\begin{tabular}{|c|c|c|c|c|c|c|}
\hline \multirow[t]{2}{*}{ Autor/Ano } & \multirow[t]{2}{*}{ Título } & \multirow{2}{*}{ Revista } & \multicolumn{4}{|c|}{ Base de dados } \\
\hline & & & PubMed & SciELO & G.A & Lilacs \\
\hline $\begin{array}{l}\text { ORNELL et al., } \\
(2020)\end{array}$ & $\begin{array}{ll}\text { Pandemia } & \text { de medo } \mathrm{e} \\
\text { Covid-19: impacto na } \\
\text { saúde mental } & \mathrm{e} \\
\text { possíveis estratégias. } & \end{array}$ & $\begin{array}{l}\text { Repositório Institucional } \\
\text { da UNB - Departamento } \\
\text { de Farmácia }\end{array}$ & & & & 01 \\
\hline $\begin{array}{l}\text { SILVA; SANTOS } \\
(2020)\end{array}$ & $\begin{array}{l}\text { Saúde do idoso em } \\
\text { tempos de pandemia } \\
\text { covid-19: cuidados de } \\
\text { enfermagem }\end{array}$ & $\begin{array}{l}\text { Revista JRG de Estudos } \\
\text { Acadêmicos }\end{array}$ & & 01 & & \\
\hline $\begin{array}{l}\text { COSTA et al., } \\
(2020)\end{array}$ & $\begin{array}{l}\text { Covid-19: seus } \\
\text { impactos clínicos e } \\
\text { psicológicos na } \\
\text { população idosa / covid- } \\
\text { 19: seus impactos } \\
\text { clínicos e psicológicos } \\
\text { na população idosa }\end{array}$ & $\begin{array}{l}\text { Revista Brazilian Journal } \\
\text { of development }\end{array}$ & 01 & & & \\
\hline $\begin{array}{l}\text { BATELLO et al., } \\
\text { (2020) }\end{array}$ & $\begin{array}{l}\text { Cuidadores de } \\
\text { em situação } r \text { de } \\
\text { pandemia: reflexos } \\
\text { sobre o cuidar e ser } \\
\text { cuidado. }\end{array}$ & Revista ABEN & & & & 01 \\
\hline $\begin{array}{l}\text { VENTURINI; } \\
\text { KINALSKI; } \\
\text { BENETTI, (2020) }\end{array}$ & 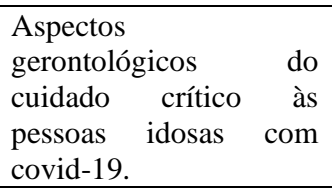 & Revista ABEN & & & & 01 \\
\hline $\begin{array}{l}\text { HAMMERSCHM } \\
\text { IDT; SANTANA, } \\
(2020)\end{array}$ & $\begin{array}{l}\text { Saúde do } \text { idoso em } \\
\text { tempos de } \\
\text { Covid-19 }\end{array}$ & Revista Cogitare & & & 01 & \\
\hline $\begin{array}{l}\text { GAZOS; GAZOS } \\
(2020)\end{array}$ & $\begin{array}{l}\text { Diagnóstico } \text { de } \\
\text { enfermagem ao idoso } \\
\text { vítima de covid-19. }\end{array}$ & $\begin{array}{l}\text { Revista Anais do } \\
\text { Congresso de Geriatria e } \\
\text { Gerontologia } \\
\text { UNIFACIG }\end{array}$ & & & 01 & \\
\hline $\begin{array}{l}\text { BARBOSA et al., } \\
(2021)\end{array}$ & $\begin{array}{l}\text { O protagonismo da } \\
\text { enfermagem no cuidado } \\
\text { ao idoso em tempos de } \\
\text { covid- } 19 \text {. }\end{array}$ & $\begin{array}{l}\text { Revista Brazilian Journal } \\
\text { of development }\end{array}$ & 01 & & & \\
\hline $\begin{array}{l}\text { SILVA et al., } \\
(2021)\end{array}$ & $\begin{array}{lr}\text { Assistência } & \text { de } \\
\text { enfermagem a } & \text { idosos } \\
\text { com Covid-19: revisão } \\
\text { escopo }\end{array}$ & $\begin{array}{l}\text { Revista Brazilian Journal } \\
\text { of development }\end{array}$ & 01 & & & \\
\hline
\end{tabular}

Fonte: Autores (2021).

\subsection{A pandemia da Covid-19}

A Severe Acute Respiratory Syndrome Coronavirus-2 (Sars-Cov-2) é uma doença que age através da infecção em diversos níveis no sistema respiratório e no organismo humano, chegando ao mais agudo que leva ao comprometimento do pulmão e consequente falta de ar, levando ao óbito. Em decorrência da falta de estudos sobre a doença, e formas de combate o vírus tornou-se uma severa pandemia mundial, alterando a rotina dos hospitais e da sociedade (Barbosa et al., 2021).

Esta pandemia é um dos maiores desafios de saúde em todo o mundo neste século. Por se tratar de uma doença nova, com conhecimento científico insuficiente sobre ela, sua rápida disseminação e sua capacidade de causar a morte de pessoas vulneráveis, é incerto quais as melhores medidas a serem tomadas para combatê-la em diferentes partes do mundo (Batello et al., 2020, Bitencourt; Andrade, 2021). 
No Brasil, o desafio é enorme, pois em um contexto de severa desigualdade social, as pessoas sabem pouco sobre todas as características do covid-19, alguns locais não possuem saneamento básico, não possuem saneamento básico e em situações de superlotação, como exemplo favelas, destaca-se ainda que a equipe de saúde além de líder com as convencionais lotações médicas e mais variadas doença, passou a trabalhar com intensidade na luta pela vida (Costa et al., 2020).

Todos os países adotaram medidas de contenção, como manutenção do distanciamento social, isolamento, bloqueio e uso de máscaras e máscaras faciais. Todas essas medidas são consideradas estratégicas para o controle de doenças populacionais. Embora essas medidas sejam parcialmente eficazes, a alta taxa de mortalidade é inconsistente. Segundo dados do Ministério da Saúde, o número acumulado de mortes em 2021 é de 586.851, colocando o Brasil em quinto lugar no ranking mundial (Barbosa et al., 2021).

Após a introdução da covid-19 no Brasil, as autoridades locais de saúde de diferentes departamentos administrativos (Governo Federal, Governos Estaduais e Municipais) tomaram uma série de medidas para controlar e prevenir a doença. Esses padrões variam de região para região, mas todas as autoridades escolheram a mesma medida de distância social. (Barbosa, 2020). Em segundo lugar, a maioria dos governantes opta por estimular essa medida e adotar estratégias de controle populacional, como o fechamento de escolas, creches e universidades, áreas comerciais não essenciais e áreas de lazer públicas. Portanto, grande parte da população aderiu ao movimento quarentenário com o objetivo de prevenir doenças e cooperar com o declínio da curva de infecção (Gazos; Gazos, 2020).

Assim, deve-se compreender que a abrangência da pandemia ocasionou uma mudança de paradigma na forma de se viver, e serem realizados contatos entre profissionais de saúde e pacientes, os idosos por serem parte da população mais afetada pelo vírus tiveram restringidas as suas poucas interações sociais com familiares e sociedade, tomados de um temor pela morte, é nesse sentido que compreender a atuação do enfermeiro nesse contexto, e os mais diversos da covid-19 em pacientes idosos torna-se tão importante.

\subsection{Idosos enquanto população de risco}

O índice de casos com covid-19 destaca-se primordialmente por atingir a um grupo de risco, dentre eles estão pacientes com doenças crônicas como diabetes, hipertensão, asma, DPOC, transplante ou quimioterapia, doença renal ou diálise, obesidade (IMC $\geq 40$ ), fumantes e idosos. 60 anos, gestantes, puérperas e crianças menores de 5 anos, homens com idade avançada e algumas morbidades anteriores parecem estar relacionados à mortalidade de pacientes hospitalizados por COVID-19. Nesse contexto, o crescimento acelerado do envelhecimento brasileiro tem ocorrido com a importante escala e impacto das doenças crônicas e infecciosas (Hammerschimidt; Santana, 2020).

É sabido que o isolamento entre os idosos é preocupante, pois apresentam maior risco de problemas cardiovasculares, neurocognitivos, autoimunes e de saúde mental. Obviamente, o distanciamento social estabelecido pela Organização Mundial da Saúde (OMS) e vigente no país afeta o comportamento dos cidadãos, sejam eles idosos ou não, isso afeta o metabolismo de todos os organismos e regula os mais diversos fatores biológicos sistêmicos, e ainda atingindo aspectos fisiológicos e psicológicos, por isso é muito importante que os profissionais de saúde saibam avaliar como o vírus afeta o metabolismo dos idosos e as consequências físicas e psicológicas (Ornel et al., 2020).

Destaca-se que no Brasil, o primeiro registro da doença foi feito no final de dezembro de 2019 e dias depois foi declarada emergência de saúde pública internacional. Desde então, a doença se desenvolveu rapidamente, enfraquecendo a capacidade de resposta dos sistemas de saúde de muitos países, incluindo o Brasil, e produzindo intervenções que mudam significativamente a vida diária das pessoas (Silva et al., 2021).

Essas medidas também afetam os trabalhadores de saúde de duas maneiras: interferem em sua vida privada e familiar por estarem sujeitos a regras de controle de doenças que valem para todos e sobrecarregam o sistema e os serviços de saúde. 
Registros de poluição, doença, morte, suicídio, ansiedade e ataques de pânico e deterioração de doenças estão se tornando mais frequentes (Silva; Santos, 2020).

Devido ao novo coronavírus, $36 \%$ dos idosos brasileiros que ainda trabalham para complementar sua renda não foram pagos ou sua renda caiu significativamente. Entre os que não têm vínculo empregatício, esse número subiu para 55\%. A crise atual causada pela pandemia global COVID-19 teve um grande impacto na economia de um país e, neste caso, também teve um grande impacto na economia global. Tudo o que aconteceu estava além do plano da empresa e das pessoas comuns (Venturini; Kinalski; Benetti, 2020).

No contexto desta pandemia, as adversidades aumentam em todas as áreas. Segundo o SEBRAE (2020), com o encerramento do comércio e de várias outras empresas, como medida preventiva contra o vírus, tem atingido não apenas a questão da saúde, mas, também da qualidade de vida, interação social, aumento da pobreza, e nesse contexto os idosos fazem parte do grupo populacional no qual sofre com isolamento devido à distância de seus familiares, amigos e de seu convívio social, impostas pelo Ministério da Saúde para prevenção da doença (Silva; Santos, 2020).

Assim, os idosos encontram-se classificados como população de risco pois, a taxa de mortalidade pelo covid-19 é bastante assombrosa neste público, pois estes são mais sujeitos à morbimortalidade. Desta, forma deve-se compreender como a enfermagem atua nesse contexto e classifica o impacto da pandemia na saúde dos idosos.

\subsection{O enfermeiro da identificação dos impactos da pandemia ao paciente idoso}

Diante dos resultados da pesquisa, deve-se destacar a importância da enfermagem durante a pandemia de Covid-19, visto que esta categoria profissional tem a capacidade de proporcionar à sua população uma cultura e sociedade pautadas nos princípios clínicos, biológicos, humanitários e de saúde. Porém, nesta crise de saúde e na conjuntura nacional, a prática da assistência, enfermagem e hospitalidade, principalmente a população idosa, tem se tornado desafiadora para os enfermeiros (Barbosa et al., 2021).

Tendo em vista a particularidade proporcionada pela Covid-19, esse grupo está sempre vulnerável ao longo do processo de envelhecimento, seja em termos de fatores biológicos, sociais, culturais e psicológicos. Sendo assim, a enfermagem tornou-se a protagonista da atenção à saúde do idoso, colocando em prática sua enfermagem clínica, preventiva e educativa, visa melhorar as condições de vida e a qualidade de vida do idoso, além de contribuir para uma melhor mudança do quadro pandêmico (Batello et al., 2020).

Logo, pode-se comprovar que neste período da pandemia de Covid-19, o cuidado ao idoso é fundamental para a população idosa, evidenciando assim todos os seus protagonistas. Esses profissionais devem readequar suas práticas e ações para atender o idoso de forma qualificada e segura (Rozenel, 2020; Alexandre; Nunes, 2020). Os autores supracitados apontaram ainda que dada a complexidade do processo de envelhecimento, suas características próprias, aliadas à alta incidência de doenças crônicas e seus efeitos, é óbvio que atenção especial deve ser dada aos idosos, incluindo prevenção, tratamento e reabilitação, que é característica do processo de trabalho da enfermagem (Hammerschmidt; Santana, 2020).

Durante a pandemia, além da complexidade do processo de envelhecimento humano, tornam-se evidentes as necessidades especiais de cuidados com os idosos, por apresentarem características e especialidades próprias. Portanto, os profissionais de enfermagem ganham destaque por atuarem em diferentes aspectos do atendimento à multidão, seja por meio da promoção e prevenção da saúde, monitoramento, cuidado profissional, etc (Meier et al., 2020). Apesar disso, a doença infecciosa de Covid-19 é mais uma prova dos idosos, o que também mostra que essa patologia está se agravando e a taxa de mortalidade é maior (Costa et al., 2020).

Portanto, o cuidado e a assistência devem ser mais efetivos e integrais, por meio do diagnóstico de enfermagem adotado pelo enfermeiro, para que receba o cuidado, busque encontrar problemas específicos e encontre ações adequadas para 
garantir resultados significativos e no combate à Covid-19, visando melhorar a qualidade de vida dos idosos, pois estes fazem parte da população suscetível e que requer o apoio de equipes multidisciplinares de saúde (Zhang, 2020). Além disso, é importante observar que durante a pandemia de Covid-19, a população idosa faz parte do grupo de risco para contrair o coronavírus, portanto, medidas como o isolamento social têm sido pautadas como principal estratégia para evitar a infecção nessas populações (Gazos; Gazos, 2020).

Desde então, os idosos ficaram mais tempo em casa. Portanto, Ornel et al., (2020), em sua pesquisa, foi enfatizado que nos casos em que o cuidado deve ser realizado no domicílio, se possível, o profissional enfermeiro deve realizar uma avaliação para verificar se o ambiente é adequado para a continuidade do cuidado. Também é determinado que é necessário estabelecer comunicação entre o enfermeiro ou equipe de saúde e o paciente e familiares para apurar as reais necessidades e fornecer orientações e cuidados necessários (ABEn, 2020).

Além disso, os pacientes e seus familiares precisam estar atentos às medidas básicas de prevenção e controle de infecções, como higiene pessoal, uso de máscaras, lavagem das mãos, uso de álcool gel 70\%, etc., para poderem cuidar pessoas. Quando houver suspeita de infecção por Covid19, seja o mais seguro possível para evitar que a infecção se espalhe para os membros da família, assim a pandemia de Covid-19 tem vários efeitos sobre a saúde, a vida social, familiar e econômica da população idosa (Silva et al., 2021).

No entanto, esse grupo possui uma série de peculiaridades que os tornam vulneráveis às doenças causadas pelo novo coronavírus e prejudicam o âmbito familiar. Portanto, é muito importante que a equipe médica seja qualificada não só para prestar uma assistência integral e humanizada aos pacientes, mas também para prestar um atendimento integral e humanizado a toda a sua família, mas também é frustrante pelas inúmeras críticas sociais que tem. surgiu a partir dele (Silva; Santos, 2020).

No entanto, considerando as ideias da autora acima, fica claro que os profissionais de enfermagem podem ter um papel preponderante no enfrentamento desse fenômeno, considerando a importância de sua atribuição preventiva, por meio da educação em saúde e da intervenção e do cuidado aos acometidos pela doença. os idosos e suas famílias (WHO, 2020). Diante dessa pandemia, os profissionais de enfermagem procuram formular diretrizes de gestão da segurança para o paciente e para os próprios profissionais, pois ele não será acometido pela doença e não ficará infectado pelo vírus (Silva; Santos, 2020).

A enfermagem oferece assistência humanizada e individualizada de acordo com as necessidades de cada paciente, tendo como padrão o diagnóstico e a intervenção de enfermagem. Para aumentar a segurança e a disseminação do vírus Covid19, os profissionais citados utilizaram equipamentos de proteção individual (EPIs), tais como: máscaras N95, chapéus, cadarços, óculos, luvas de látex, aventais longos descartáveis e rosto máscaras (LIU et al., 2020). Use água e sabão para limpar as mãos correta e rigorosamente. Use álcool 70\% para desinfetar as mãos e evitar o contato com os olhos, nariz e boca (Batello et al., 2020).

Em vista do acima exposto, mostra a importância do papel dos enfermeiros nos cuidados de saúde dos idosos durante a pandemia de Covid-19. Eles prometeram desempenhar um papel no cuidado, gestão e melhor cuidado dos idosos em resposta à Covid -19. Enfatize a melhoria de sua saúde e qualidade de vida. Além de desempenhar suas funções, os profissionais também se preocupam com sua biossegurança para garantir sua saúde e não a disseminação de vírus.

\section{Conclusão}

A pandemia tem um impacto significativo na saúde dos idosos e estimula a necessidade de proteção, respeito, cuidado, dignidade e redes de apoio, mas também expõe a discriminação por idade, julgamento e ridículo. As operações de proteção à distância devem manter a autonomia e independência do idoso, e os profissionais precisam ter um conhecimento específico desse grupo de pessoas. No momento da pandemia, intensifica-se a demanda por um cuidado estável, qualificado e 
seguro ao idoso, por meio da formação profissional básica, é necessário ter novos significados para as ações de cuidado ao idoso, respeitar a diversidade e olhar para as condições presentes e futuras.

Vale destacar que, a atuação da enfermagem no idoso de rua tem trazido muita reflexão e aprendizado, principalmente no desenvolvimento de ferramentas que garantam o cuidado a esse grupo social. As diversas ações realizadas para garantir a avaliação do estado de saúde dos idosos, o acesso aos serviços de saúde, os procedimentos de saneamento e as condições de habitação durante a pandemia têm reforçado a possibilidade e o potencial da intervenção do enfermeiro, bem como despertado e reforçado a compreensão da situação e ainda sobre os aspectos da heterogeneidade atribuída para os idosos.

Os profissionais de enfermagem devem reformular suas práticas e ações para atender a população idosa de forma qualificada e segura, e nesse período trabalharam com os idosos para a promoção e promoção e prevenção de saúde, monitoramento, cuidado e atenção especial em todas as áreas, isso inclui facilitar o tratamento e a reabilitação em situações mais complexas. Imediatamente.

Além de promover a saúde da família, a enfermeira também mantém um vínculo pautado na comunicação constante com os idosos e suas famílias, principalmente por meio do uso de tecnologia, gerando responsabilidade, pois fornece informações sobre medicação, cuidado, orientação aos familiares e, por fim, fornece assistência ao paciente nas mais diversas situações de isolamento social.

Como resultado, eles são desacreditados nas práticas de julgamento, discriminação por idade e exclusão, especialmente aquelas experimentadas durante a pandemia. Com o surgimento da pandemia covid-19, o desenvolvimento e a prática de planos de emergência são fundamentais para a proteção de idosos vulneráveis, mas é importante ressaltar que políticas públicas isoladas não garantem isso.

Portanto, recomenda-se que outros estudos sejam realizados sobre a temática para discutir aspectos práticos desses impactos, levando em consideração a importância da qualidade de vida do idoso e do impacto positivo da enfermagem e suas ferramentas de atuação na rotina do idoso.

\section{Referências}

Alexandre, L. B. S. P., \& Nunes, M. I (2020). Problematização sobre a pandemia da COVID-19 como auxílio na formação de enfermeiras/os. Revista Nursing. 23(266), 4294-4300. http://dx.doi.org/10.36489/nursing.2020v23i266p4294-4307.

Associação Brasileira de Enfermagem (ABEn Nacional) (2020). Departamento Científico de Enfermagem Gerontológica. Comunicação aos trabalhadores de enfermagem das instituições de longa permanência de idosos (ILPI) para o enfrentamento da disseminação da COVID-19. ABEn Nacional [Internet].

Barbosa, I. R (2020). Incidência e mortalidade por COVID-19 na população idosa brasileira e sua relação com indicadores contextuais: um estudo ecológico. Revista Brasileira de geriatria gerontologia, 23(1), 56-98. http://dx.doi.org/10.1590/1981-22562020023.200171.

Barbosa, M. M. A., Duarte, R. B., Nascimento, C. E. M., Costa, J. B Lima, L. R., Freitas \& Brandão, M. R. F. S (2021). O protagonismo da enfermagem no cuidado ao idoso em tempos de covid-19. Revista Brazilian Journal of Development, 7(8), 34-46. https://doi.org/10.34117/bjdv7n8-292.

Batello, G. V. V. A. T., Guimarães, M. S. A., Pereira, I. A. C., Bandeira, M \& Nunes, D. P (2020). Cuidadores de idosos em situação de pandemia: reflexos sobre o cuidar e ser cuidado. Revista ABEn, 19(6), 20-25.

Bitencourt, S. M., \& Andrade, C. B (2021). Trabalhadoras da saúde face à pandemia: por uma análise sociológica do trabalho de cuidado. Revista Ciência \& Saúde Coletiva da Associação Brasileira de Saúde Coletiva. 26, 3. 1013-1022. http://dx.doi.org/10.1590/1413-81232021263.42082020.

Brasil (2020 a). Ministério da Saúde. Protocolo de manejo clínico para o novo coronavírus (2019-nCoV). Ministério da Saúde

Brasil (2020 b). Ministério da Saúde. Secretaria de Vigilância em Saúde. Boletim Epidemiológico Especial COE-COVID-19 (16). Ministério da Saúde. https://www.saude.gov.br/images/pdf/2020/May/21/2020-05-19---BEE16---Boletim-do-COE13h.pdf.

Costa, F. A., Silva, A. S., Oliveira, C. B. S., Costa, L. C. S., Paixão, M. E. S., Celestino, M. N. S., Araújo, M. C., Azevedo, S. M. A., Silva, C. R. C., Santos, I. L \&V. L (2020). Covid-19: seus impactos clínicos e psicológicos na população idosa/covid-19: seus impactos clínicos e psicológicos na população idosa. Revista Brazilian Journal of Development, 6(7), 245-254. https://doi.org/10.34117/bjdv6n7-580.

Estrela, C. (2018). Metodologia Científica: Ciência, Ensino, Pesquisa. Editora Artes Médicas. 
Research, Society and Development, v. 10, n. 14, e145101422054, 2021

(CC BY 4.0) | ISSN 2525-3409 | DOI: http://dx.doi.org/10.33448/rsd-v10i14.22054

Gazos, W. M. J \& Gazos, S. M (2020). Diagnóstico de enfermagem ao idoso vítima de covi-19. Revista Anais do Congresso de Geriatria e Gerontologia da UNIFACIG, 1(1), 78-89.

Hammerschidt, K. S. A \& Santana, R. F (2020). Saúde do idoso em tempos de pandemia Covid-19. Revista Cogitare, 25(3), 30-40. http://dx.doi.org/10.5380/ce.v25i0.72849.

Liu, K., Chein, Y., Lin, R \& Han, K (2020). Clinical features of COVID-19 in elderly patients: A comparison with young and middle-aged patients. J Infect 10.1016/j.jinf.2020.03.005.

Meier, J. G. Cabral, L. P A., Zanesco, C., Grden, C. R. B., Fadel, C. B.., Bordin, D (2020) Factors associated with the frequency of medical consultations by older adults: a national study. Rev. esc. enferm. USP, 54. https://doi.org/10.1590/S1980-220X2018048103544.

Ornell, F., Schuch, J. B., Sordi, A. O., \& Kesssler, F H. P (2020). Pandemia de medo e Covid-19: impacto na saúde mental e possíveis estratégias. Repositório Institucional da UNB - Departamento de Farmácia, artigo de conclusão, p. 15.

Pereira A. S. et al. (2018). Metodologia da pesquisa científica. UFSM.

Rozenek, M. (2020). Definición de caso COVI-19: ¿Debe ser la misma para el adulto mayor? Rev. Hosp Ital. B. Aires. 40(2), 58-59.

Silva, D. C., Donoso, M. T. V., Barbosa, J. A. G \& Ferreira, D.F (2021). Assistência de enfermagem a idosos com Covid-19: revisão de escopo. Revista Brazilian Journal of Development, 4(3), 77-89. https://doi.org/10.34119/bjhrv4n3-359.

Silva, M. P. P. E \& Santos, W. L (2020). Saúde do idoso em tempos de pandemia covid-19: cuidados de enfermagem. Revista JRG De Estudos Acadêmicos, 3(7), 214-223. https://doi.org/10.5281/zenodo.4118417.

Venturini, L., Kinalski, S. S \& Benetti, E. R (2020). Aspectos gerontológicos do cuidado crítico às pessoas idosas com covid-19. Revista ABEn, 19(6), 55-60.

World Health Organization (WHO) (2020). State of the world's nursing 2020: investing in education, jobs and leadership. WHO., 2020. https://apps.who.int/iris/bitstream/handle/10665/331677/9789240003279-eng.pdf?sequence=1\&isAllowed=y.

Zhang, W. Manual de Prevenção e Controle da Covid-19 segundo o Doutor Wenhong Zhang. PoloBooks. 